\title{
Out-of-time-ordered correlators and the Loschmidt echo in the quantum kicked top: How low can we go?
}

\author{
Sreeram PG, ${ }^{1}$ Vaibhav Madhok, ${ }^{1}$ and Arul Lakshminarayan ${ }^{1}$ \\ ${ }^{1}$ Department of Physics, Indian Institute of Technology Madras, Chennai, India 600036
}

\begin{abstract}
The out-of-time-ordered correlators (OTOC) and the Loschmidt echo are two measures that are now widely being explored to characterize sensitivity to perturbations and information scrambling in complex quantum systems. Studying few qubits systems collectively modelled as a kicked top, we solve exactly the three- and four- qubit cases, giving analytical results for the OTOC and the Loschmidt echo. While we may not expect such few-body systems to display semiclassical features, we find that there are clear signatures of the exponential growth of OTOC even in systems with as low as 4 qubits in appropriate regimes, paving way for possible experimental measurements. We explain qualitatively how classical phase space structures like fixed points and periodic orbits have an influence on these quantities and how our results compare to the large-spin kicked top model. Finally we point to a peculiar case at the border of quantum-classical correspondence which is solvable for any number of qubits and yet has signatures of exponential sensitivity in a rudimentary form.

PACS numbers:
\end{abstract}

\section{INTRODUCTION}

The contemporary interest and progress in quantum information processing have happened along with control over single or few particle systems that are driving home the novelty of unique quantum phenomena such as entanglement. It has also opened doors for investigation in the time domain, with exquisite control of individual quantum systems in the laboratory and the ability to drive these systems with designer Hamiltonians that can simulate phenomena as diverse as many-body-localization to ergodicity, chaos and thermalization. Two experiments that preserve the coherence and purity of complex manybody time-evolving states illustrate the richness of this domain [1, 2].

The first of these [1 involved the study of 3 qubits in a superconducting transmon setup that simulated the quantum kicked top. Using state tomography they made connections between the onset of chaos and concomitant enhancement in the entanglement. The second 2 involved a two-dimensional Bose-Einstein condensate of ${ }^{87} \mathrm{Rb}$ atoms, implementing effectively a 6 particle BoseHubbard Hamiltonian. The study of thermalization via the development of entanglement in such experiments on isolated quantum systems is of interest in the foundations of statistical mechanics, and they test the Ergodic Thermalization Hypothesis (ETH) that is currently of great theoretical interest as well. Connections between low-dimensional ergodicity and chaos with entanglement, general quantum correlations and state tomography have long been studied, mostly theoretically, (for example in [3-18]), although a cold-atom experiment as early as 2009 [19] was a pioneering work in this direction.

These experiments also beg the question of how statistical properties such as thermalization and semiclassical properties such as chaos manifest in such low-dimensional quantum systems. The 3 -qubit transmon experiment is based on the mapping of the well-studied quantum kicked top to a many-spin Floquet system. However, while traditional studies of quantum chaos are for large spin $j$ [20], this experiment involved only $j=3 / 2$ and the mapped system is in fact a nearest neighbor transverse field Ising model which is integrable. In any case, the solvability of this as well as the $j=2$ system which involves nonintegrable next-nearest-neighbor interactions was demonstrated in 21]. Such a study did show that it is possible to see some generic features and even some random matrix theory properties in such small systems. For example, it showed how with increasing the parameter controlling the non-integrability, entanglement moves from being bipartite to multipartite, sharing it globally and demonstrating its monogamous nature.

Starting from [22] which considered just two qubits $(j=1)$ analytically and 3 qubits $(j=3 / 2)$ numerically, there have been studies that followed the fate of the few qubit kicked top [15, 21, 23. A recent experiment 24] used NMR to study the 2 qubit version of the kicked top already displaying some semiclassical features but also peculiar quantum ones such as time- and parameter- periodicity [23].

However, most of the traditional signatures of quantum chaos are based on statistical spectral properties, such as nearest-neighbor spacing statistics 20, which do not make sense for small systems. As a matter of principle, the effective Planck constant $h_{\text {eff }}$ in such systems is large and hence quantum-classical correspondence time scales, such as the Ehrenfest time of $E_{f} \sim \log \left(1 / h_{\mathrm{eff}}\right) / \lambda_{C}$, where $\lambda_{C}$ is the classical Lyapunov exponent are very short. Quantum properties such as superposition manifest rapidly in time and thus experiments that involve coherent time-evolution also seem to be out of reach of semiclassical features.

The present work is placed in this context as one that explores how two measures based on the time-evolution fare in ferreting out non-integrability and chaos out of small quantum systems that are already experimentally 
realizable, hence the question is how low can we go? These measures are the out-of-time-ordered correlator (OTOC), being intensely studied now in a remarkable variety of contexts, and the Loschmidt echo, which has a longer history of study in low-dimensional chaos. We find that although only very short-time information is available, OTOC of $j=2$ and $j=5 / 2$ kicked-tops already show definite precursors of exponential growth, and many properties of the echo are also shared by large $j$ systems, although the exponential decay may not be apparent, at least in the regimes we have addressed here. Thus the answer to the question seems to be "pretty low". We also initiate the study of a kicked top of arbitrary spin $j$, but when the chaos parameter is so "absurdly large" that the Lyapunov exponent $\lambda_{C}$ is as large as $\log \left(1 / h_{\mathrm{eff}}\right)$, and the Ehrenfest time is still of order 1! This "dual" case also manifests for low values of $j$, one does not require is a very large value of the chaoticity parameter for the top. The kicked top Floquet operator, as we shall discuss, can be written as a sum of just 4 rotations (for integer $j$ ), and hence the interactions need not be implemented at all.

An array of quantum signatures of chaos have already been studied. Fidelity decay in quantum systems [25, 26], level statistics [27, 28, properties of regular and irregular wavefunctions 29 31] and quantum scars 32, signatures in single particle billiards 33, 34, semiclassical trace formulas [35] and imprints on quantum correlations and tomography 3 5, 7, 15, 19. Recent trends that focus on many-body systems, include studies involving connections of quantum chaos to OTOCs, entropic uncertainty relations, and the rate of scrambling of quantum information in many-body systems with consequences ranging from the foundations of quantum statistical mechanics, quantum phase transitions, and thermalization on the one hand to the scrambling of information in many-body systems and black holes on the other hand [36 45].

The OTOC, in their simplest form, captures the growth of the incompatibility between two operators, when one of them is evolved in the Heisenberg picture while the other is stationary [36, 39, 46, 50. From the commutator-Poisson bracket connection, this gives an analog of the classical separation of two trajectories with quantum mechanical operators replacing the classical phase space trajectory. For two Hermitian observables, OTOC is given by

$$
C_{W, V}(\tau)=-\left\langle[W(x, \tau), V(y, 0)]^{2}\right\rangle,
$$

where the local operators $W$ and $V$ act on sites $x$ and $y$ respectively and $W(x, \tau)=U^{\dagger}(\tau) W(x, 0) U(\tau)$ is the Heisenberg evolution of operator $W$ under unitary dynamics $U(\tau)$. The expectation value is taken with respect to the thermal state at some temperature which we take to be infinite. In sufficiently chaotic systems, the OTOC essentially vanishes till the information of the operator perturbation at $x$ reaches $y$, during which phase the operator becomes highly nonlocal, an occurrence that is dubbed operator scrambling. Thereafter there is a rapid increase of the OTOC before it saturates in a finite system at which stage the localized information at $x$ is considered to have been scrambled throughout the system, and it is not possible to recover it from any local subset. If the rapid increase of the OTOC is exponential $\sim e^{2 \lambda_{Q} t}$, $\lambda_{Q}$ has been referred to as a quantum Lyapunov exponent.

If the system has a bound spectrum this implies instability in a finite space and can be taken as a definition of quantum chaos. Thinking of systems with a well-defined semiclassical limit, note that simple systems such as the inverted parabolic potential $-x^{2}$ have trivially exponentially growing OTOC, but are of course not chaotic, but merely unstable. Similarly, there could be naturally isolated unstable orbits in an otherwise integrable system and special operators may still show exponential OTOC growth. Still, the jury is out on the role of OTOC in general and hence studying them in as many scenarios is of interest. Systems with well-defined semiclassical or classical limits are of special interest as it is well understood in what sense they are non-integrable and what the classical Lyapunov exponents are, and there have been several studies on this [51 57], including some on the quantum kicked top 58,60

Though quantum systems do not show sensitivity to perturbations in initial state vectors, integrable and chaotic quantum systems show remarkably different behavior and sensitivity when the system dynamics itself is perturbed 61, 62. One of the concepts used to capture this notion of quantum chaos is the Loschmidt echo that is related to the fidelity between the evolution of a quantum system with exact dynamics and propagation under a slightly perturbed Hamiltonian 63 65. Alternatively, this quantifies the distance between the forward propagation of a system and its time-reversed dynamics under small perturbations. This is interesting as the question of time-reversal itself and its connections to chaos, both quantum and classical, has been one of the foundational questions in physics. The debate around the microscopic origins of the second law of thermodynamics from underlying time-reversal invariant classical mechanics leads to interesting paradoxes. For example, could one reverse the momenta of all particles in a system causing the entropy to decrease thereby violating the second law 61]? In this work, our focus is to study Loschmidt echo for a few qubit kicked top that is exactly solvable [21.

Loschimidt echo is defined as

$$
F(t)=\left|\left\langle\phi\left|e^{i H^{\prime} t} e^{-i H t}\right| \phi\right\rangle\right|^{2},
$$

where $H$ is the Hamiltonian for the forward evolution and $H^{\prime}$ is the perturbed Hamiltonian representing imperfect time reversal, i.e., the Hamiltonian responsible for backward evolution. The perturbed evolution can be due to environmental noise and thus there is an intimate connection between Loschmidt echo and decoherence 66 The Loschmidt echo has a rather complex behavior that depends on the state $|\phi\rangle$, the nature of the Hamiltonian $H$-whether it is integrable or not, the degree of chaos if it 
is not integrable and also on the strength of the perturbation that defines $H^{\prime}$. In certain regimes, an exponential decay of the fidelity has been observed with a rate that is the classical Lyapunov exponent.

Recently the question of sensitivity to perturbations is connected to the accuracy and robustness of quantum information processing devices. After all, the quantum device/simulator is a many-body complex quantum system and one needs to benchmark its accuracy [59, 67, 68. How does one trust a quantum simulator that invariably involves a many-body chaotic Hamiltonian with a rapid proliferation of errors, especially near a quantum critical point that is typically characterized by high entanglement/complexity and a large Schmidt rank of the system density matrix 69 71]? While these questions have been under active research for many decades, only recently experiments have reached the level of sophistication and control where non-integrability, chaos, and thermalization of closed quantum systems are studied by manipulating individual interacting quantum bits. Another interesting avenue on the applications of Loschmidt echo is the application to quantum-limited metrology and making sensors. Since chaotic systems are sensitive to perturbations, this suggests a way to for high precision metrology 72 .

This paper is organized as follows. In Sec. (II) the kicked top is introduced and some of its classical properties are mentioned. A complete solution of the quantum problem for 3 and 4 qubit cases is also carried out, in the sense that explicit expressions for the powers of the Floquet operator are given in terms of the Chebyshev polynomials. In Sec. (III) the OTOC is derived for the 3 and 4 qubit kicked tops and their dependence on time and the chaoticity parameter is discussed. The OTOC is also compared with that for a larger number of spins, found numerically. The peculiar case when the number of spins is arbitrary by the chaos parameter is very large is also discussed in this section. In Sec. (IV), the Loschmidt echo is discussed and we summarise and discuss future directions in Sec. (V).

\section{THE CASE OF KICKED TOP}

The quantum kicked top is characterized by the angular momentum vector $\left(J_{x}, J_{y}, J_{z}\right)$, and the Hamiltonian 20, 73, 74, is given by

$$
H=\frac{\kappa_{0}}{2 j} J_{z}^{2} \sum_{n=-\infty}^{\infty} \delta(t-n \tau)+\frac{p}{\tau} J_{y}
$$

It consists of rotations and impulsive rotations caused by periodic kicks at regular intervals of time $\tau$. The time evolution of the top is given by the unitary operator

$$
\mathcal{U}=\exp \left[-i\left(\kappa_{0} / 2 j \hbar\right) J_{z}^{2}\right] \exp \left[-i(p / \hbar) J_{y}\right]
$$

which describes the evolution from one kick to the next. Angle of rotation about the $y$ axis is given by $p$, and $\kappa_{0}$ is (a) $\kappa_{0}=\pi / 2$

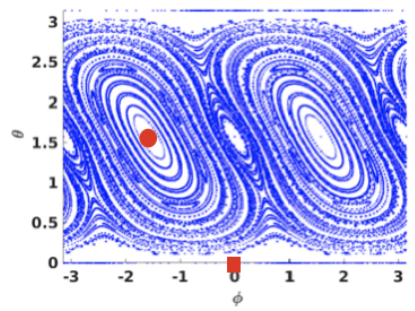

(c) $\kappa_{0}=3 \pi / 2$

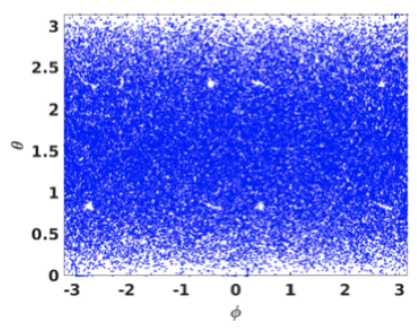

(b) $\kappa_{0}=\pi$

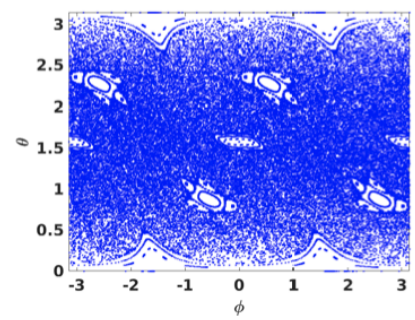

(d) $\kappa_{0}=2 \pi$

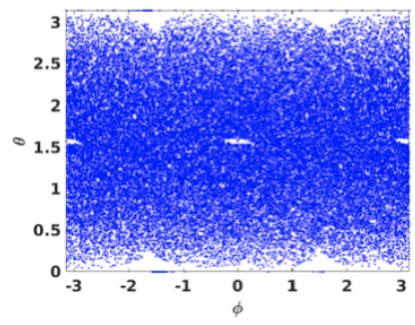

FIG. 1: (a) Regular (b,c) mixed phase space and (d) chaotic phase space resulting from the classical kicked top dynamics. Points labeled with red square and red circle in a) correspond to initial states $\Theta=0, \Phi=0$ on a period- 4 orbit and $\Theta=$ $\pi / 2, \Phi=-\pi / 2$ at the center of regular island respectively.

the chaoticity parameter, which is a measure of the twist applied between kicks. Here we set $\hbar=1$ and $p=\pi / 2$. In the limit of very large angular momentum, the classical limit is reached. $i^{t h}$ iteration the classical map of the unit sphere phase space $X_{i}^{2}+Y_{i}^{2}+Z_{i}^{2}=1$ onto itself is given by

$$
\begin{aligned}
X_{i} & =Z_{i-1} \cos \left(\kappa_{0} X_{i-1}\right)+Y_{i-1} \sin \left(\kappa_{0} X_{i-1}\right) \\
Y_{i} & =-Z_{i-1} \sin \left(\kappa_{0} X_{i-1}\right)+Y_{i-1} \cos \left(\kappa_{0} X_{i-1}\right) \\
Z_{i} & =-X_{i-1}
\end{aligned}
$$

where $X_{i}, Y_{i}, Z_{i}=J_{x, y, z} / j$

Dynamics of a particle under these equations are simulated numerically for different initial states: $\left(X_{0}, Y_{0}, Z_{0}\right)$, and for two strengths of the chaos, $\kappa_{0}=0.5$ and 2.5, as shown in Fig. 1] conventionally termed as regular and mixed phase space structures respectively. For $\kappa_{0}=0$ the classical map is integrable, being just a rotation, but for $\kappa_{0}>0$ chaotic orbits appear in the phase space, and when $\kappa_{0}>6$ it is essentially fully chaotic. Connection to a many-body model can be made by considering the large $\mathbf{J}$ spin as the total spin of spin $=1 / 2$ qubits, replacing $J_{x, y, z}$ with $\sum_{l=1}^{2 j} \sigma_{l}^{x, y, z} / 2$ [75, 76]. The Floquet operator is then that of $2 j$ qubits, an Ising model with all-to-all homogeneous coupling and a transverse magnetic field:

$$
\mathcal{U}=\exp \left(-i \frac{\kappa_{0}}{4 j} \sum_{l<l^{\prime}=1}^{2 j} \sigma_{l}^{z} \sigma_{l^{\prime}}^{z}\right) \exp \left(-i \frac{\pi}{4} \sum_{l=1}^{2 j} \sigma_{l}^{y}\right)
$$

Here $\sigma_{l}^{x, y, z}$ are the standard Pauli matrices, and an overall phase is neglected. The case of 2-qubits, $j=1$, has 
been analyzed in 22] wherein interesting arguments have been proposed for the observation of structures not linked to the classical limit. In this case, several quantum correlation measures were also calculated in $[23$. For $j=3 / 2$, the three-qubit case is a nearest neighbor kicked transverse Ising model, known to be integrable [10, 77]. For higher values of the spin, the model maybe considered few-body realizations of non-integrable systems. In general only the $2 j+1$ dimensional permutation symmetric subspace of the full $2^{2 j}$ dimensional space is relevant to the kicked top.

\section{A. Solving the the 3 and 4 qubit kicked tops}

The solutions in these cases were discussed first in [21], where a wide variety of entanglement measures, from entropy to concurrence were studied and compared with available experimental data. We recount here the essential details of the solutions for the sake of a self-contained narrative. First, there is the general observation of an "up-down" or parity symmetry:

$$
\left[\mathcal{U}, \otimes_{l=1}^{2 j} \sigma_{l}^{y}\right]=0
$$

valid for any number of qubits. It is therefore optimal to work with a basis that is both permutation symmetric and is adapted to the parity.

For $j=3 / 2$ or the 3 -qubit case, the standard 4dimensional spin-quartet permutation symmetric space $\{|000\rangle,|W\rangle=(|001\rangle+|010\rangle+|100\rangle) / \sqrt{3},|\bar{W}\rangle=(|110\rangle+$ $|101\rangle+|011\rangle) / \sqrt{3},|111\rangle\}$ is parity symmetry adapted to form the basis

$$
\begin{aligned}
\left|\phi_{1}^{ \pm}\right\rangle & =\frac{1}{\sqrt{2}}(|000\rangle \mp i|111\rangle), \\
\left|\phi_{2}^{ \pm}\right\rangle & =\frac{1}{\sqrt{2}}(|W\rangle \pm i|\bar{W}\rangle) .
\end{aligned}
$$

In this basis the Floquet unitary operator is given by

$$
\mathcal{U}=\left(\begin{array}{cc}
\mathcal{U}_{+} & 0 \\
0 & \mathcal{U}_{-}
\end{array}\right)
$$

where 0 is the $2 \times 2$ null matrix, and $2 \times 2$-dimensional blocks $\mathcal{U}_{+}$and $\mathcal{U}_{-}$are written in the bases $\left\{\phi_{1}^{+}, \phi_{2}^{+}\right\}$and $\left(\left\{\phi_{1}^{-}, \phi_{2}^{-}\right\}\right)$, and are referred to as positive and negative)parity subspaces in our discussion. We have,

$$
\mathcal{U}_{ \pm}= \pm e^{\mp \frac{i \pi}{4}} e^{-i \kappa}\left(\begin{array}{cc}
\frac{i}{2} e^{-2 i \kappa} & \mp \frac{\sqrt{3}}{2} e^{-2 i \kappa} \\
\pm \frac{\sqrt{3}}{2} e^{2 i \kappa} & -\frac{i}{2} e^{2 i \kappa}
\end{array}\right)
$$

corresponding to parity eigenvalue \pm 1 . For simplicity the parameter $\kappa=\kappa_{0} / 6$ is used in these expressions.

Expressing Eq. 10 as a rotation $\left(e^{-i \theta \sigma^{\hat{\eta}}}\right)$ by angle ' $\theta$ ' about an arbitrary axis $(\hat{\eta}=\sin \alpha \cos \beta \hat{x}+\sin \alpha \sin \beta \hat{y}+$ $\cos \alpha \hat{z})$, and a phase, we obtain, $\cos \theta=\frac{1}{2} \sin 2 \kappa, \beta=$ $\pi / 2+2 \kappa$, and $\sin \alpha=\sqrt{3} /(2 \sin \theta)$. Thus the time evolution is the propagator which is simply the power $\mathcal{U}^{n}$ is block-diagonal with blocks $\mathcal{U}_{ \pm}^{n}$, which are explicitly given by,

$$
\mathcal{U}_{ \pm}^{n}=( \pm 1)^{n} e^{-i n\left( \pm \frac{\pi}{4}+\kappa\right)}\left(\begin{array}{cc}
\alpha_{n} & \mp \beta_{n}^{*} \\
\pm \beta_{n} & \alpha_{n}^{*}
\end{array}\right)
$$

where,

$$
\begin{aligned}
\alpha_{n} & =T_{n}(\chi)+\frac{i}{2} U_{n-1}(\chi) \cos 2 \kappa \\
\beta_{n} & =(\sqrt{3} / 2) U_{n-1}(\chi) e^{2 i \kappa} .
\end{aligned}
$$

Here the Chebyshev polynomials $T_{n}(\chi)$ and $U_{n-1}(\chi)$ of the first and second kinds are used and are defined as

$$
T_{n}(\chi)=\cos (n \theta) \quad U_{n-1}(\chi)=\sin (n \theta) / \sin \theta,
$$

with $\chi=\cos \theta=\sin (2 \kappa) / 2=\sin \left(\kappa_{0} / 3\right) / 2$. Hence the matrix elements of the time $n$ propagator are explicitly given by polynomials of order $n$ in the variable $\sin \left(\kappa_{0} / 3\right)$.

We further present an exact solution to a kicked top with spin $j=2$, modelled using four qubits, where each qubit is coupled to every other qubit by the same strength. Hamiltonian for such a system can be easily obtained from Eq. 3. by substituting $j=2$. It is particularly interesting to study a four-qubit kicked top as this is the smallest system where all-to-all interaction among qubits is different from that of nearest-neighbor interaction. Similar to that of the three-qubit kicked top, we are again confined to $(2 j+1)$-dimensional permutation symmetric subspace of the total $2^{2 j}$-dimensional Hilbert space. In this case the parity symmetry reduced and permutation symmetric basis in which $\mathcal{U}$ is block-diagonal is

$$
\begin{aligned}
\left|\phi_{1}^{ \pm}\right\rangle & =\frac{1}{\sqrt{2}}(|W\rangle \mp|\bar{W}\rangle), \\
\left|\phi_{2}^{ \pm}\right\rangle & =\frac{1}{\sqrt{2}}(|0000\rangle \pm|1111\rangle), \\
\left|\phi_{3}^{+}\right\rangle & =\frac{1}{\sqrt{6}} \sum_{\mathcal{P}}|0011\rangle_{\mathcal{P}}
\end{aligned}
$$

where $|W\rangle=\frac{1}{2} \sum_{\mathcal{P}}|0001\rangle_{\mathcal{P}},|\bar{W}\rangle=\frac{1}{2} \sum_{\mathcal{P}}|1110\rangle_{\mathcal{P}}$, and $\sum_{\mathcal{P}}$ sums over all possible permutations.

A peculiarity of 4 -qubits is that $\left|\phi_{1}^{+}\right\rangle$is an eigenstate of $\mathcal{U}$ with eigenvalue -1 for all values of the parameter $\kappa_{0}$. Thus the $5-$ dimensional space splits into $1 \oplus 2 \oplus 2$ subspaces on which the operators are $\mathcal{U}_{0}=-1$ and $\mathcal{U}_{ \pm}$. In this basis, the Floquet unitary operator $\mathcal{U}$ becomes block diagonal, which makes it easy to write the $n^{\text {th }}$ power of the unitary operator $\mathcal{U}$ as

$$
\mathcal{U}^{n}=\left(\begin{array}{ccc}
\mathcal{U}_{0}^{n} & 0_{1 \times 2} & 0_{1 \times 2} \\
0_{2 \times 1} & \mathcal{U}_{+}^{n} & 0_{2 \times 2} \\
0_{2 \times 1} & 0_{2 \times 2} & \mathcal{U}_{-}^{n}
\end{array}\right)
$$

This simplifies the problem significantly. Various blocks are written here explicitly, we have

$$
\mathcal{U}_{0}=\left\langle\phi_{1}^{+}|\mathcal{U}| \phi_{1}^{+}\right\rangle=-1
$$


which is a part of the positive-parity subspace. Block $\mathcal{U}_{+}$ written in the $\left\{\phi_{2}^{+}, \phi_{3}^{+}\right\}$basis, is

$$
\mathcal{U}_{+}=-i e^{-\frac{i \kappa}{2}}\left(\begin{array}{cc}
\frac{i}{2} e^{-i \kappa} & \frac{\sqrt{3} i}{2} e^{-i \kappa} \\
\frac{\sqrt{3} i}{2} e^{i \kappa} & -\frac{i}{2} e^{i \kappa}
\end{array}\right)
$$

while $\mathcal{U}_{-}$in the basis $\left\{\phi_{1}^{-}, \phi_{2}^{-}\right\}$, is

$$
\mathcal{U}_{-}=e^{-\frac{3 i \kappa}{4}}\left(\begin{array}{cc}
0 & e^{\frac{3 i \kappa}{4}} \\
-e^{-\frac{3 i \kappa}{4}} & 0
\end{array}\right)
$$

where $\kappa=\kappa_{0} / 2$.

In a manner similar to the case of 3 -qubits above, the time $n$ propagator is now written compactly in terms of the Chebyshev polynomials. We have

$$
\mathcal{U}_{+}^{n}=e^{-\frac{i n(\pi+\kappa)}{2}}\left(\begin{array}{cc}
\alpha_{n} & i \beta_{n}^{*} \\
i \beta_{n} & \alpha_{n}^{*}
\end{array}\right)
$$

where

$$
\begin{aligned}
& \alpha_{n}=T_{n}(\chi)+\frac{i}{2} U_{n-1}(\chi) \cos \kappa \\
& \beta_{n}=\frac{\sqrt{3}}{2} U_{n-1}(\chi) e^{i \kappa}
\end{aligned}
$$

with $\chi=\sin \kappa / 2=\sin \left(\kappa_{0} / 2\right) / 2$. The negative parity subspace evolution operator is

$$
\mathcal{U}_{-}^{n}=e^{-\frac{3 i n \kappa}{4}}\left(\begin{array}{cc}
\cos \frac{n \pi}{2} & e^{\frac{3 i \kappa}{4}} \sin \frac{n \pi}{2} \\
-e^{-\frac{3 i \kappa}{4}} \sin \frac{n \pi}{2} & \cos \frac{n \pi}{2}
\end{array}\right) .
$$

Although for simplicity we use the same symbols $\alpha_{n}$ and $\beta_{n}$ for the propagator matrix entries in the 3 and 4 qubit cases, they are not the same. However, in either case, we note the important identity that $\left|\alpha_{n}\right|^{2}+\left|\beta_{n}\right|^{2}=1$, following from the unitarity of the propagators involved, arises from the Pell equation for the Chebyshev polynomials:

$$
T_{n}(x)^{2}+\left(1-x^{2}\right) U_{n-1}^{2}=1 .
$$

\section{OTOC AND THE KICKED TOP}

The out-of-time-ordered correlators (OTOC) are closely connected to the growth of the incompatibility of observables due to the dynamics. They are currently being studied in a wide variety of contexts from many-body physics to field theories, quantum gravity, and black holes in a remarkable coming together of many research communities. They are thought of as a way to investigate the "quantum butterfly effect", which was also the role and motivation for the introduction of the Loschmidt echo. Both of these quantities, in systems with a semiclassical limit, have regimes where the classical Lyapunov exponent plays a role: as (half) the rate of the exponential growth of OTOC and as the rate of exponential decay of the echo. The Lyapunov exponent may be seen more clearly in the OTOC as the echo has a rather complex dependence on the perturbation used, however recent works have pointed out explicit connections between OTOC in an averaged sense and the echo [78.

Let $A(0)$ be some observable and let $A(t)=\mathcal{U}^{-t} A(0) \mathcal{U}^{t}$ be its Heisenberg time evolution. We define the OTOC as

$$
C_{\rho}(t)=-\frac{1}{2} \operatorname{Tr}\left(\rho[A(t), A(0)]^{2}\right) .
$$

where $\rho$ is some state of the system. In particular we deal with the infinite temperature state $\rho=I /(2 j+1)$ denote the corresponding OTOC as $C_{\infty}(t)$. The phrase "outof-time-ordered" is justified for these quantities as the commutator contains terms such as $\langle A(t) A(0) A(t) A(0)\rangle$ wherein the operators are not monotonically ordered in time. OTOC have been used an indicator of information scrambling as some initially localized operator or "information" in a many-body system gets entangled with other one-particle operators on other sites and leads to a complex state wherein the initial information is practically lost. For nonintegrable chaotic systems, especially with a semiclassical limit, the expected exponential growth of the OTOC

$$
C_{\rho}(t) \sim e^{2 \lambda_{Q} t}
$$

has been observed and the quantum Lyapunov exponent $\lambda_{Q}$ has been found to be close to the classical one. The exponential growth is observed till the log-time or the Ehrenfest time which scales as $\ln (1 / h) / \lambda_{C}$ where $h$ is a scaled Planck's constant and $\lambda_{C}$ the classical Lyapunov exponent.

The kicked top has been previously used in OTOC studies such as in [58, 59] and variations of it that break the permutation symmetry are beginning to be studied as well as potential models of "holography" 60 as well as from the point of view of experimental realizations via NMR for example. Previous studies of the kicked top OTOC were in the semiclassical limit of large $j$, wherein only numerical results are accessible. It is of interest to ask how these properties manifest themselves in the solvable highly quantum regime of small $j$ which are accessible to present day experiments. We are limited by short time scales and the exponential growths cannot be clearly observed in these cases. Yet it is intriguing to have exactly solvable cases wherein we may see such growth in a rudimentary form and study the transition to semiclassical regimes. Due to our restriction to the permutation symmetric subspace, it is not possible to use a single qubit operator and we take the symmetric subspace projection of the collective spin variable $A(0)=\sum_{i=1}^{2 j} \sigma_{i}^{z} / 2=J_{z}$ as the observable.

\section{A. OTOC in 3 qubits: $j=3 / 2$}

For $j=3 / 2$, the 3 qubit case, this restriction takes the form of $J_{z}=(3 / 2)|000\rangle\langle 000|-(3 / 2)| 111\rangle\langle 111|+$ 
$(1 / 2)|W\rangle\langle W|-(1 / 2)| \bar{W}\rangle\langle\bar{W}|$. Using the basis in Eq. $[7]$ in which the time evolution further block-diagonalizes and noting that $J_{z}\left|\phi_{1}^{ \pm}\right\rangle=(3 / 2)\left|\phi_{1}^{\mp}\right\rangle, \quad J_{z}\left|\phi_{2}^{ \pm}\right\rangle=$ $(1 / 2)\left|\phi_{2}^{\mp}\right\rangle$, we use

$$
J_{z}=\left(\begin{array}{cc}
0_{2 \times 2} & S \\
S & 0_{2 \times 2}
\end{array}\right), \quad S=\frac{1}{2}\left(\begin{array}{ll}
3 & 0 \\
0 & 1
\end{array}\right) .
$$

This leads to

$$
J_{z}(n)=\mathcal{U}^{-n} J_{z} \mathcal{U}^{n}=\left(\begin{array}{cc}
0 & \mathcal{U}_{+}^{-n} S \mathcal{U}_{-}^{n} \\
\mathcal{U}_{-}^{-n} S \mathcal{U}_{+}^{n} & 0
\end{array}\right)
$$

Considering the case of the infinite temperature OTOC $C_{\infty}(n)$, we separate it as

$$
C_{\infty}(n)=C_{2}(n)-C_{4}(n),
$$

where $C_{2}(n)=\operatorname{Tr}\left[J_{z}^{2}(n) J_{z}(0)^{2}\right] / 4$ is the two-point correlator and $C_{4}(n)=\operatorname{Tr}\left[J_{z}(n) J_{z}(0) J_{z}(n) J_{z}(0)\right] / 4$ is the four-point correlator which is out-of-time ordered. This leads to

$$
\begin{aligned}
& C_{2}(n)=\frac{1}{4}\left[\operatorname{Tr}\left(\mathcal{U}_{+}^{-n} S^{2} \mathcal{U}_{+}^{n} S^{2}\right)+\operatorname{Tr}\left(\mathcal{U}_{-}^{-n} S^{2} \mathcal{U}_{-}^{n} S^{2}\right)\right] \\
& C_{4}(n)=\frac{1}{4}\left[\operatorname{Tr}\left(\mathcal{U}_{+}^{-n} S \mathcal{U}_{-}^{n} S\right)^{2}+\operatorname{Tr}\left(\mathcal{U}_{-}^{-n} S \mathcal{U}_{+}^{n} S\right)^{2}\right] .
\end{aligned}
$$

Plugging in the elements of $\mathcal{U}_{ \pm}^{n}$ from Eq. 111 and simplifications lead to

$$
\begin{aligned}
& C_{2}(n)=\frac{1}{16}\left(41-32\left|\beta_{n}\right|^{2}\right) \\
& C_{4}(n)=(-1)^{n} \frac{1}{16}\left(41-160\left|\beta_{n}\right|^{2}+128\left|\beta_{n}\right|^{4}\right),
\end{aligned}
$$

where $\beta_{n}$ is given by Eq. $12 \mathrm{~b}$, and hence

$$
\left|\beta_{n}\right|^{2}=\frac{3}{4} U_{n-1}^{2}\left[\frac{1}{2} \sin \left(\frac{\kappa_{0}}{3}\right)\right] .
$$

For small $\kappa_{0}$ when the dynamics is near-integrable these give

$$
C_{\infty}(n) \approx\left\{\begin{array}{l}
\frac{1}{6} n^{2} \kappa_{0}^{2}-\frac{13}{2592} n^{4} \kappa_{0}^{4} n \text { even } \\
\frac{5}{8}+\frac{1}{288}\left(n^{2}-1\right)^{2} \kappa_{0}^{4} n \text { odd }
\end{array}\right.
$$

This shows a marked odd-even behaviour with the even time OTOC increasingly quadratically with time at the lowest order. The odd-even effect is quite easily understood as for very small $\kappa_{0}$ the dynamics is essentially one of rotation about the $y$ axis by $\pi / 2$ and hence the $J_{z}$ operator with a concentration in the $z$ direction is rotated practically to its negative at times $2 \bmod 4$ and to itself at times $0 \bmod 4$ and hence almost commutes, but at times $1 \bmod 4$ or $3 \bmod 4$ is concentrated on the $y$ and $-y$ directions and maximally fails to commute. Indeed the constant term $5 / 8$ is nothing but $-\operatorname{Tr}\left[J_{y}, J_{z}\right]^{2} / 4=$ $\operatorname{Tr} J_{x}^{2} / 4$. A quadratic growth has also been observed in the Hadamard quantum walk [79] and we may expect a general power-law growth of the OTOC to be a general integrable and near-integrable feature [51, 80, that we see in this small and solvable system exactly.

Now we turn attention to fixed and small times but for arbitrary values of the parameter $\kappa_{0}$. It follows from Eq. 27) and Eq. 29) that $C_{\infty}(1)=5 / 8$ irrespective of the value of $\kappa_{0}$, as $U_{0}(x)=1$. This shows no interesting dynamical behaviour and the OTOC have a diffusive time scale over which the properties depend on the observable chosen as well. The next time steps already are of interest:

$$
\begin{aligned}
& C_{\infty}(2)=6 \sin ^{2}\left(\kappa_{0} / 3\right)\left(1-\frac{3}{4} \sin ^{2}\left(\kappa_{0} / 3\right)\right) \\
& C_{\infty}(3)=\frac{5}{8}+18 \sin ^{4}\left(\kappa_{0} / 3\right)\left(1-\frac{1}{2} \sin ^{2}\left(\kappa_{0} / 3\right)\right)^{2},
\end{aligned}
$$

$C_{\infty}(n)$ being a polynomial of order $4(n-1)$ in $\chi=$ $\sin \left(\kappa_{0} / 3\right) / 2$. The curves for $C_{\infty}(2)$ and $C_{\infty}(3)$ are shown

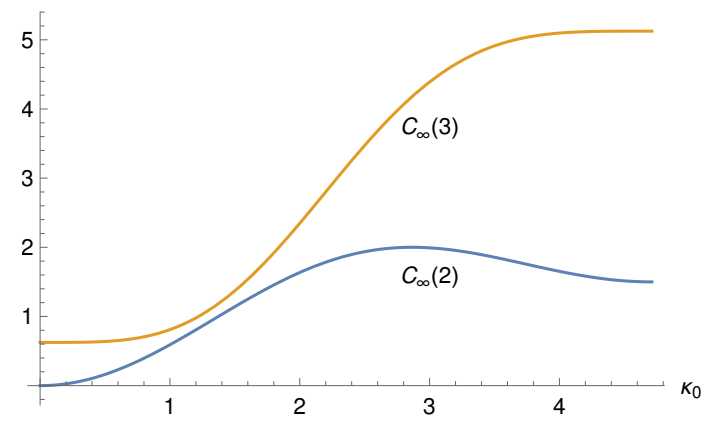

FIG. 2: The OTOC for the 3 qubit kicked top at times 2 and 3 as a function of the chaos parameter $\kappa_{0}$. In all figures the observable used is $J_{z}$. Note the difference in the behavior around $\kappa_{0}=0$, the near-integrable regime and also that the increase is monotonic at time 3 , and reaches a maximum at $\kappa_{0}=3 \pi / 2$, when the top is essentially already fully chaotic.

in Fig. (2) for convenience and we see that they increase with $\kappa_{0}$ and $C_{\infty}(3)$ is monotonically increasing over the entire range of interest $\kappa_{0} \in[0,3 \pi / 2]$, reaching the maximum value at $\kappa_{0}=3 \pi / 2$. A more global view is provided in Fig. (3) where the OTOC for $j=3 / 2$ shown as a function of the time, split into even and odd ones, and the parameter $\kappa_{0}$. There would be a periodicity beyond the value of $\kappa_{0}=3 \pi / 2$, which provides an interesting boundary. Exactly at this point, the classical dynamics is fairly chaotic and we do see a sharp increase in the OTOC values for short times even in this small $j$ value.

To give an indication of the growth, $\ln \left[C_{\infty}(n)\right]$ is plotted in Fig. (4) for $1 \leq n \leq 3$. This has just three points, but the trend is clear and we may even interpret this as signs of the exponential growth of the OTOC that one expects in chaotic systems. To compare this with higher values of $j$, we show in Fig. (5) the case for some larger values of the spin $j$, but with $\kappa_{0}=3 \pi / 2$ in all the cases. We do see an increase and saturation in the slope with 

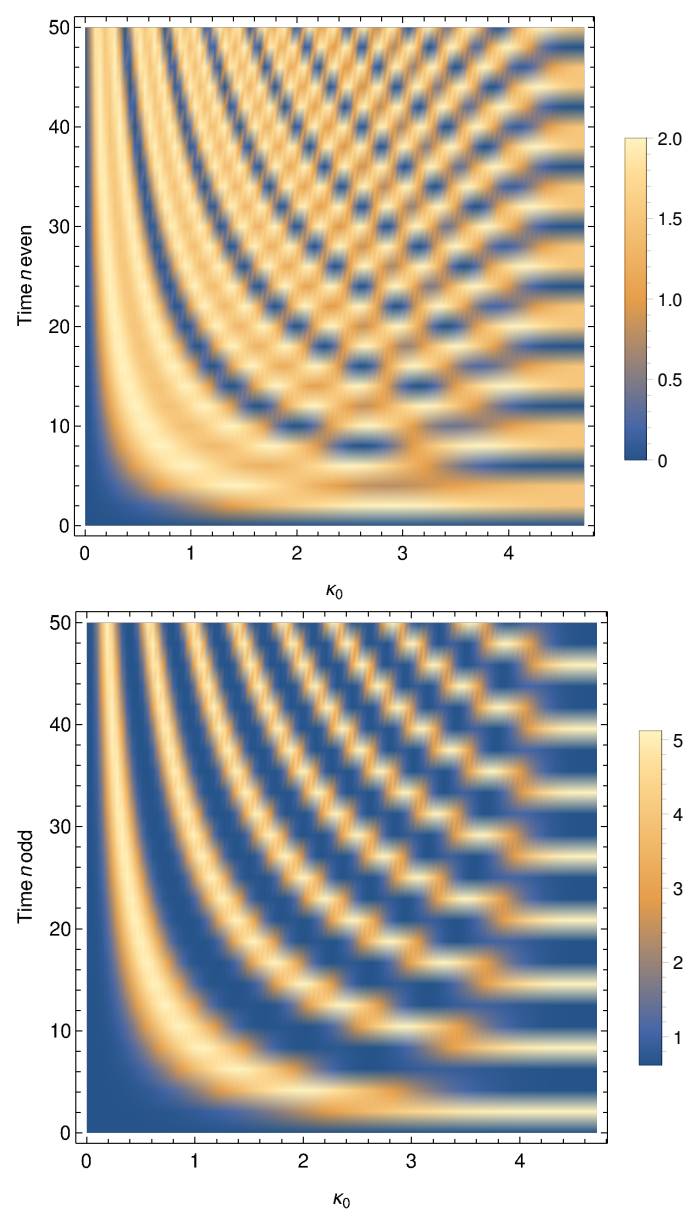

FIG. 3: A density plot of the OTOC as function of time and the parameter $\kappa_{0}$ for 3 qubits, it is separated for even and odd times for reasons explained in the text.

increasing $j$ values. It is interesting to observe from the same figure that with $j=5 / 2$ the OTOC slope has already saturated and hence at this value of the parameter, while $j=3 / 2$ is too low, $j=5 / 2$ may be just enough. To explore this further we turn to the other solvable case of $j=2$ and compare it with higher values of $j$, as well as study the peculiar case of $\kappa_{0}=\pi j$ for arbitrary $j$.

\section{B. OTOC in 4 qubits, $j=2$, and the peculiar case of $\kappa_{0}=\pi j$ for arbitrary $j$.}

The 4 qubit case we reiterate can be qualitatively different from the case of 3 as it has next-nearest neighbor interactions and is a rudimentary non-integrable model. The calculations do not pose a serious problem as the unitary time evolution is still block-diagonalized into utmost 2-dimensional spaces, see Eq. (15). the equations get a little bit more involved, but nevertheless can be exactly solved, especially with the help of computer algebra. Skipping the details, we present the final results again separating the cases of different time parities. For

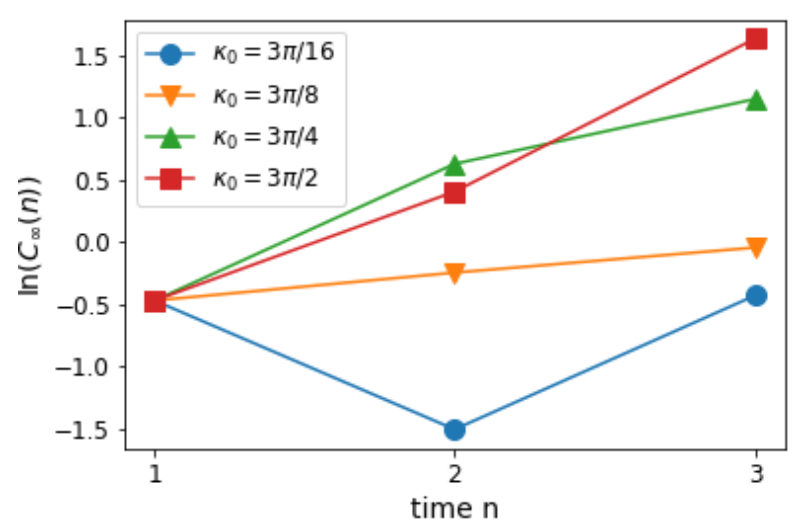

FIG. 4: The OTOC is shown in linear-log scale for a few values of $\kappa_{0}$. The dynamics is predominantly chaotic at $\kappa_{0}=3 \pi / 2$ and is reflected in what appears to be a near linear OTOC growth.

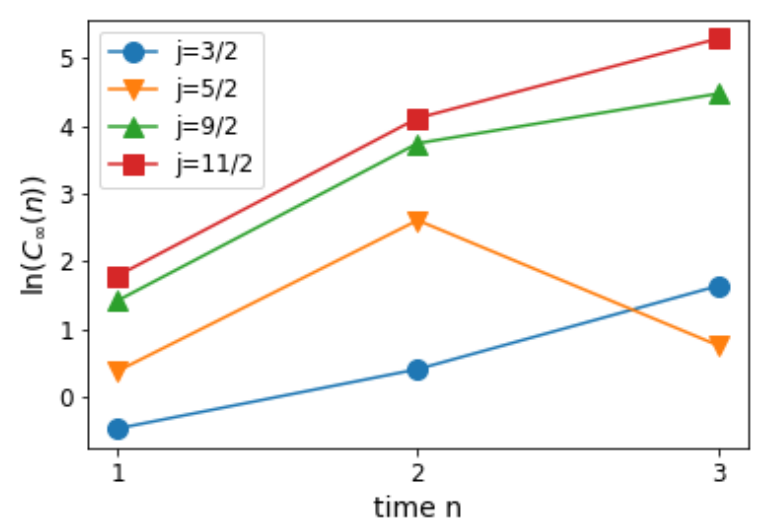

FIG. 5: The OTOC in the linear-log scale, when $\kappa_{0}=3 \pi / 2$ and the $j$ value is increased. The slope at $j=5 / 2$ already is well-saturated to those corresponding to larger $j$ values.

time $n$ even we get

$$
\begin{aligned}
C_{\infty}(n)= & \frac{1}{5}\left[34-16\left|\beta_{n}\right|^{2}\right. \\
& \left.-32 \operatorname{Re}\left(\alpha_{n}^{2} e^{i n \kappa_{0} / 4}\right)-2 \cos \left(3 n \kappa_{0} / 4\right)\right],
\end{aligned}
$$

and for odd $n$,

$$
\begin{aligned}
C_{\infty}(n)=\frac{1}{5}[25 & -16\left|\beta_{n}\right|^{2} \\
& \left.-16(-1)^{(n-1) / 2} \operatorname{Im}\left(\alpha_{n} e^{i n \kappa_{0} / 2}\right)\right] .
\end{aligned}
$$

Here the $\alpha_{n}$ and $\beta_{n}$ involve the Chebyshev polynomials and are from Eq. 20a. It follows that $C_{\infty}(1)=1$ irrespective of $\kappa_{0}$. Expressions for short times maybe 
explicitly extracted and for $n=2,3$ are

$$
\begin{aligned}
& C_{\infty}(2)=\frac{1}{5}\left(28-30 \cos \left(\kappa_{0} / 2\right)+6 \cos \left(\kappa_{0}\right)-4 \cos \left(3 \kappa_{0} / 2\right)\right) \\
& C_{\infty}(3)=\frac{1}{10}\left(37-36 \cos \left(\kappa_{0}\right)+9 \cos \left(2 \kappa_{0}\right)\right) .
\end{aligned}
$$

While $C_{\infty}(2)$ is a monotonically increasing function for $0 \leq \kappa_{0} \leq 2 \pi$ and is a maximum at $\kappa_{0}=2 \pi, C_{\infty}(3)$ vanishes at this point having a maximum at $\kappa_{0}=\pi$. These special values of $\kappa_{0}$ correspond to $\pi j$ and $\pi j / 2$. Notice that for $j=3 / 2, C(3)$ was a maximum at $\kappa_{0}=\pi j$ (see Fig. (2)), this difference between half-integer angular momenta and integer ones persists, and such features have also been noticed in entanglement before [21.

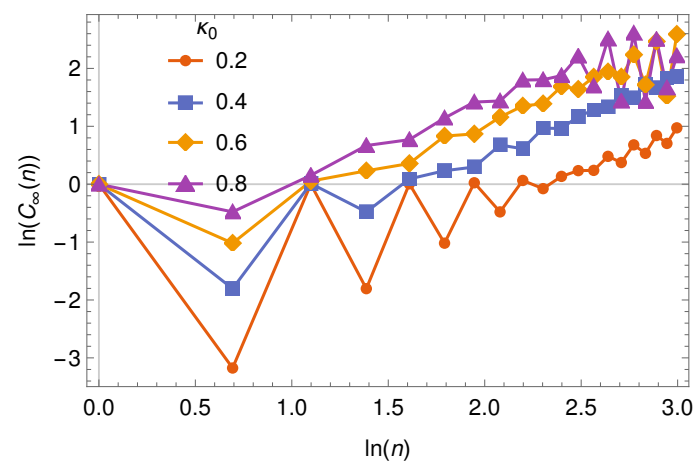

FIG. 6: The 4 qubit OTOC growth in log-log scale for values of $\kappa_{0}$ when the dynamics is near-integrable. The growths are consistent with power-laws, taking into account the odd-even features in time.

For relatively small values of $\kappa_{0}$, when the classical system is near-integrable there is modest OTOC growth mostly governed by power laws as shown in Fig. (6). At large values of $\kappa_{0}$, the OTOC grows rapidly, as seen in Fig. (7) and then oscillates in an apparently irregular manner. Of special interest again is $\kappa_{0}=2 \pi$, be-

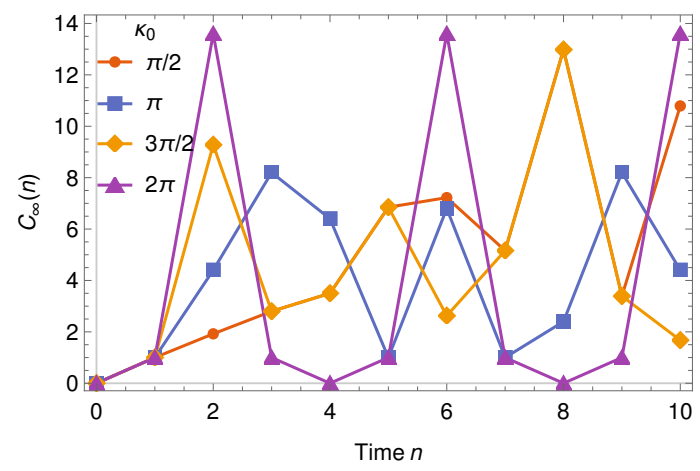

FIG. 7: The 4 qubit OTOC for larger values of $\kappa_{0}$, the large growth at $\kappa_{0}=2 \pi$ is to be noted along with its periodicity.

yond which there is a symmetric behavior equivalent to a smaller value of $\kappa_{0}$ and hence certainly not reflecting any semi-classical property. For this case, it is amusing that

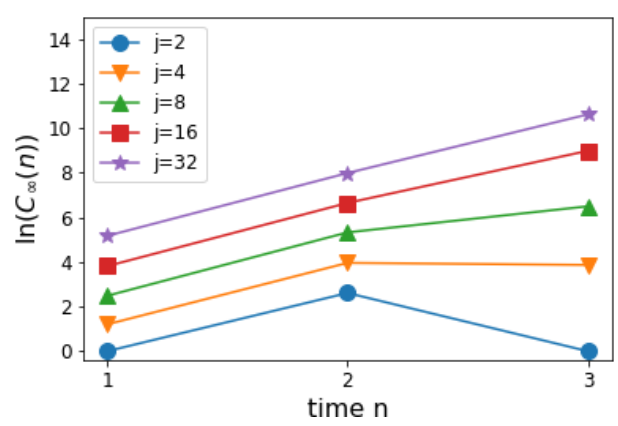

FIG. 8: The $j=2,4$ qubit OTOC at $\kappa_{0}=2 \pi$ compared with that of larger number of qubits, showing how the initial growth spurt is already reflecting the semiclassical Lyapunov exponent.

the initial growth between $C_{\infty}(1)=1$ and $C_{\infty}(2)=68 / 5$, which is all that is there, in the sense that there is timesymmetry and periodicity beyond, already reflects the large $j$ growth of OTOC at $\kappa_{0}=2 \pi$. The classical dynamics is highly chaotic at this parameter value and we may expect purely exponential growth of the OTOC. This is shown in Fig. (8), where we only plot the first 3 time steps. Using the first 2 steps of the case $j=2$, we may be bold enough to find the quantum Lyapunov exponent of Eq. (24) as $0.5 \ln (68 / 5) \sim 1.3$ and compare with the classical value of $\lambda_{C}=\ln \left(\kappa_{0}\right)-1 \sim 0.84$. We note of course that the classical exponent comes from an infinite time average and the kicked top, unlike the baker's or the cat map, is not a uniformly hyperbolic system. Thus it can hardly be expected that finite-time quantum properties from a particular observable reflect this number exactly and we see that even for large $j$ the slope is not significantly changed towards the classical value. Thus it seems plausible that with only 4 qubits one can observe the exponential growth of the OTOC due to quantum chaos.

As the extreme case of $\kappa_{0}=\pi j$ registers the largest growth of the OTOC for the 3 and 4 qubit systems studied above, it is natural to investigate this for an arbitrary value of $j$. In this case the Floquet unitary operator

$$
\mathcal{U}=e^{-i \pi J_{z}^{2} / 2} e^{-i \pi J_{y} / 2}
$$

enjoys many special properties, that we intend to investigate in detail elsewhere. For integer $j$ values it is a sum of 4 pure rotations and in general, for integer $j$, we note that when $\kappa_{0}=\pi r / s$ where $r$ and $s$ are relatively prime integers,

$$
\begin{aligned}
\mathcal{U}_{r, s}= & e^{-i r \pi J_{z}^{2} / 2 s} e^{-i \pi J_{y} / 2} \\
& =\sum_{l=0}^{2 s-1} a_{l}(r, s) e^{-i \pi l J_{z} / s} e^{-i \pi J_{y} / 2}
\end{aligned}
$$


where

$$
a_{l}(r, s)=\frac{1}{2 s} \sum_{m=0}^{2 s-1} e^{-i \pi m l / s} e^{-i \pi r m^{2} / s}
$$

are Gauss sums. A similar sum over $4 s$ terms applies for half-integer $j$ values. We record them as possible routes to implementing the kicked top experimentally when $\kappa_{0}$ is some rational multiple of $\pi$, as the torsion is replaced by a sum of rotations. For the case of $j=2$, or $r=1, s=2$, we note that $\mathcal{U}^{8}=I$, where $I$ is identity. These maps remind one of the cat maps, whose quantum mechanics is exactly periodic.

For large value of $j$ we notice that the quantumclassical correspondence time, the Ehrenfest or log-time is $\sim \ln (2 j+1) / \lambda_{C}=\ln (2 j+1) / \ln (\pi j) \sim 1$. Thus we are at the true border of the correspondence and do not expect to see classical effects for times beyond a few steps, however large $j$ may be, and indeed we find that only $n=1,2$ are unique and of interest. We find remarkably simple expressions for these:

$$
\begin{aligned}
C_{\infty}(1) & =\frac{1}{6} j(j+1) \\
C_{\infty}(2) & =\frac{2}{15} j(j+1)\left(3 j^{2}+3 j-1\right),
\end{aligned}
$$

they being related to squares and $4^{\text {th }}$ powers of integers. It reassuringly returns 1 and $68 / 5$ for the case $j=2$ which we have discussed above. This results in the quantum Lyapunov exponent of $\ln \left(C_{\infty}(2) / C_{\infty}(1)\right) \sim$ $\ln (j)+0.3$ which is to be compared with the classical one $\ln (\pi j)-1 \sim \ln (j)+0.14$. Thus the principal growth of the two Lyapunov exponents are identical and we emphasize that this is in itself quite a remarkable fact. Thus while this extreme case is highly special it does reflect the large classical chaos that underlies the system. Analysis for $\kappa_{0}$ other fractions of $\pi j$ are therefore of interest.

\section{LOSCHMIDT ECHO AND THE KICKED TOP}

Loschmidt echo, as discussed above, is a quantifier of quantum chaos based on the overlap of a given state with itself when evolved by a perturbed and an exact Hamiltonian. In general, this depends on the choice of the initial state, nature and magnitude of perturbation, degree of chaos. To make the echo state independent, one can look at the decay by considering an average over initial states from Haar measure for finite dimensional systems, $\overline{F_{d}}\left(\kappa_{0}, \kappa_{0}^{\prime}, n\right)=\int d\left|\psi_{0}\right\rangle F_{d}\left(\kappa_{0}, \kappa_{0}^{\prime}, n,\left|\psi_{0}\right\rangle\right)$ and 81,82 .

$$
\overline{F_{d}}\left(\kappa_{0}, \kappa_{0}^{\prime}, n\right)=\frac{1}{d(d+1)}\left(d+\left|\operatorname{Tr}\left[\mathcal{U}^{-n}\left(\kappa_{0}\right) \mathcal{U}^{n}\left(\kappa_{0}^{\prime}\right)\right]\right|^{2}\right)
$$

where $d$ is the dimension of the Hilbert space of the states. Essentially, the echo depends on the quantity $\left|\operatorname{Tr}\left[\mathcal{U}^{-n}\left(\kappa_{0}\right) \mathcal{U}^{n}\left(\kappa_{0}^{\prime}\right)\right]\right|^{2}$, which can be calculated easily to obtain, for the three qubit kicked top,

$$
\overline{F_{3}}\left(\kappa_{0}, \kappa_{0}^{\prime}, n\right)=\frac{1}{5}\left(1+\left|\alpha_{n} \tilde{\alpha_{n}^{*}}+\beta_{n} \tilde{\beta_{n}^{*}}+\beta_{n}^{*} \tilde{\beta_{n}}+\alpha_{n}^{*} \tilde{\alpha_{n}}\right|^{2}\right)
$$

where $\tilde{\alpha_{n}}$ and $\tilde{\beta_{n}}$ are $\alpha_{n}\left(\kappa_{0}^{\prime}\right)$ and $\beta_{n}\left(\kappa_{0}^{\prime}\right)$ respectively and $\kappa_{0}^{\prime}=\kappa_{0}+\delta \kappa_{0}$. Here, $\delta \kappa_{0}$ is the strength of perturbation. For the four qubit top, this gives

$$
\overline{F_{4}}\left(\kappa_{0}, \kappa_{0}^{\prime}, n\right)=\frac{1}{30}\left(5+\mid 1+e^{\frac{i n \delta \kappa_{0}}{4}}\left(\alpha_{n} \tilde{\alpha_{n}^{*}}+\beta_{n} \tilde{\beta_{n}^{*}}+\beta_{n}^{*} \tilde{\beta_{n}}+\alpha_{n}^{*} \tilde{\alpha_{n}}+\left.2 e^{\frac{3 i n \delta \kappa_{0}}{8}}\left(\cos ^{2}(n \pi / 2)+\sin ^{2}(n \pi / 2) \cos \left(3 \delta \kappa_{0} / 8\right)\right)\right|^{2}\right)\right.
$$

Therefore, we have the exact expressions for the Loschmidt echo for the cases at hand and explore.

Figure (9) shows that when the perturbation strength is low (order of $10^{-2}$ ), we see a Gaussian (quadratic in Log-Log plot) decay for the 3 and 4 qubit kicked top respectively. Once the size of perturbation is increased, we see a departure from the quadratic decay as is shown in Fig (10). However, keeping the same perturbation strength, one observes an exponential decay in the echo as one increases the spin size for the kicked top as shown in (11). Value of $j=32$ starts showing an exponential decay as evident on a log linear scale. As one increases the perturbation strength, as in Fig. 12, one sees an exponential decay for $j=16$ and $j=32$ - a forerunner for the exponential decay that is the hallmark of Loschmidt decay in quantum chaotic systems. For large dimensional chaotic systems, as one increases the perturbation strength, there is a transisition from quadratic to exponential decay that saturates at the value given by the classical Lyapunov exponents [81. We do see an antecedent of this decay in Fig. 13 as on increasing perturbation strength, the decay rate saturates to a fixed value. Though we are still far from the semiclassical quantum regime of large $j$, these numerical results serve as a precursor of Lyapunov decay for higher dimensional quantized chaotic Hamiltonians.

\section{A. Fidelity decay for states}

In this section, by considering the example of the 3 qubit kicked top system, we demonstrate how classical 


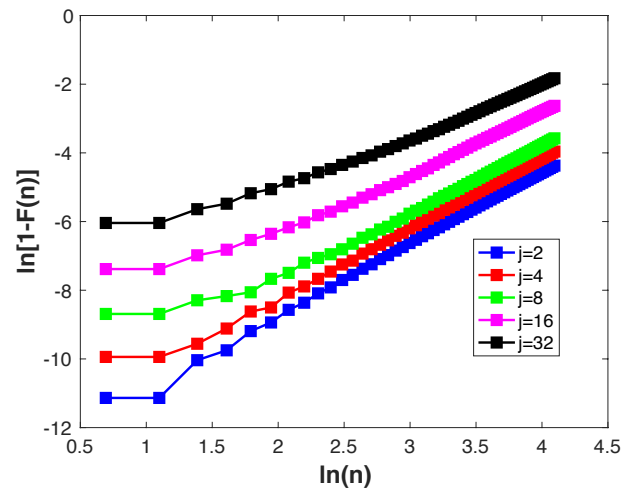

FIG. 9: The quadratic fall for the Loschmidt echo is shown with on a $\log$-log scale, when $\kappa_{0}=2 \pi$ for a few $j$ values including $j=2$. The perturbation strength is 0.01 .

phase space features have an influence on the Loschmidt echo. Analysis for four qubit states follows analogously. Three-qubit permutation symmetric initial states used are coherent states located at

$$
\begin{aligned}
X_{0} & =\sin \theta_{0} \cos \phi_{0}, \\
Y_{0} & =\sin \theta_{0} \sin \phi_{0}, \\
Z_{0} & =\cos \theta_{0},
\end{aligned}
$$

on the phase space sphere and given by [83, 84,

$$
\left|\psi_{0}\right\rangle=\left|\theta_{0}, \phi_{0}\right\rangle=\otimes^{2 j}\left(\cos \left(\theta_{0} / 2\right)|0\rangle+e^{-i \phi_{0}} \sin \left(\theta_{0} / 2\right)|1\rangle\right) .
$$

We study time evolution and fidelity decay of two completely different three-qubit states ((i) $|0,0\rangle$ and (ii) $|\pi / 2,-\pi / 2\rangle)$, shown in Fig. (1). The coherent state at $|0,0\rangle$ for three qubits is $\otimes^{3}|0\rangle$. It is on a period- 4 orbit in the classical phase space and is represented with a red square in 1. $\otimes^{3}|+\rangle_{y}$ corresponds to the coherent state at $|\pi / 2,-\pi / 2\rangle$, which is a fixed point as per regular classical phase space structure, and eventually becomes unstable as we move from regular to mixed phase space, shown by a red circle in Fig.(1). Let us consider the state on the period- 4 orbit, corresponding to the coherent state at $|0,0\rangle$ which is $\otimes^{3}|0\rangle$.

$$
\begin{gathered}
\mathcal{U}^{n}|000\rangle \equiv\left|\psi_{n}\right\rangle=\frac{1}{2} e^{-i n\left(\frac{3 \pi}{4}+\kappa\right)}\left\{( 1 + i ^ { n } ) \left(\alpha_{n}|000\rangle\right.\right. \\
\left.\left.+i \beta_{n}|\bar{W}\rangle\right)+\left(1-i^{n}\right)\left(i \alpha_{n}|111\rangle-\beta_{n}|W\rangle\right)\right\}
\end{gathered}
$$

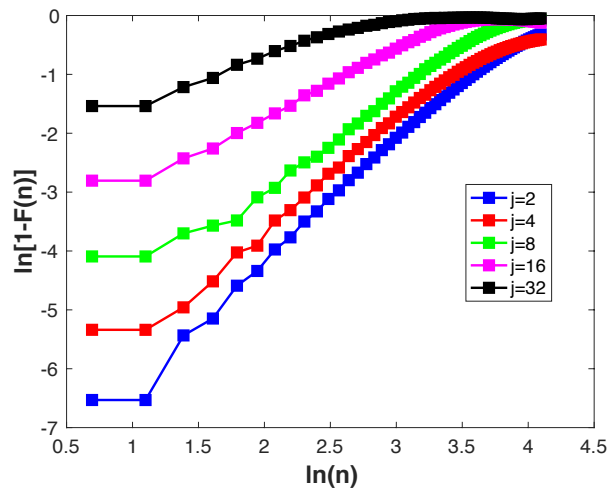

FIG. 10: The breakdown of quadratic fall for the Loschmidt echo is shown with on a $\log -\log$ scale, when $\kappa_{0}=2 \pi$ for a few $j$ values including $j=2$. The perturbation strength is 0.1 .

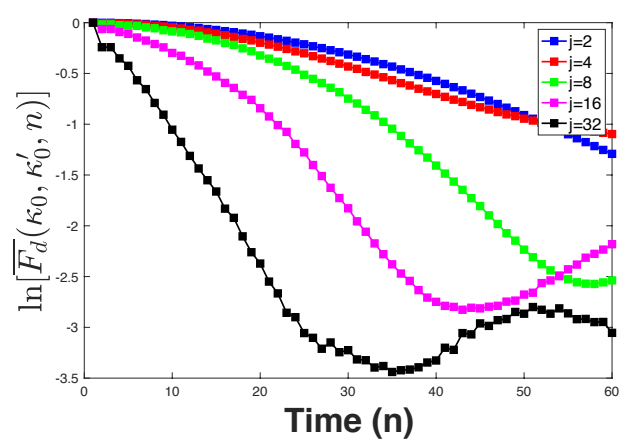

FIG. 11: Loschmidt decay on a linear log scale for some values of $j$. Perturbation strength, $\delta \kappa_{0}$, is 0.1 and $\kappa_{0}=2 \pi$. It can be seen that $j=32$ case is showing exponential decay - a forerunner of the Lyapunov decay.

Loschmidt decay can be computed by looking at the overlap of this state with another, evolved with a unitary of slightly different chaoticity parameter $\kappa_{0}^{\prime}$.

$$
\begin{aligned}
F_{3}\left(\kappa_{0}, \kappa_{0}^{\prime}, n,\left|\psi_{0}\right\rangle\right) & =\left|\left\langle 000\left|\mathcal{U}^{-n}\left(\kappa_{0}\right) \mathcal{U}^{n}\left(\kappa_{0}^{\prime}\right)\right| 000\right\rangle\right|^{2} \\
& =\left|\alpha_{n}^{*} \tilde{\alpha_{n}}+\beta_{n}^{*} \tilde{\beta_{n}}\right|^{2}
\end{aligned}
$$

Expansion in powers of $\delta \kappa_{0}$ at $\kappa_{0}=3 \pi / 2$ yields the quadratic term as the leading term that is non-zero for $n=4$ and beyond. This explains the extremely slow fall in fidelity for this state at $\kappa_{0}=3 \pi / 2$. In contrast, 


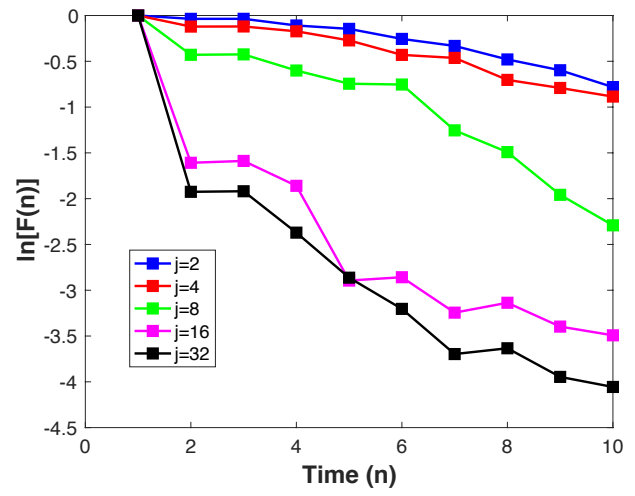

FIG. 12: Loschmidt decay on a linear log scale for some values of $j$. Perturbation strength, $\delta \kappa_{0}$, is 0.5 and $\kappa_{0}=2 \pi$. It can be seen that $j=16$ and $j=32$ case is showing exponential decay - a forerunner of the Lyapunov decay. To extract the Lyapunov exponent, one needs to go for a much larger $j$.

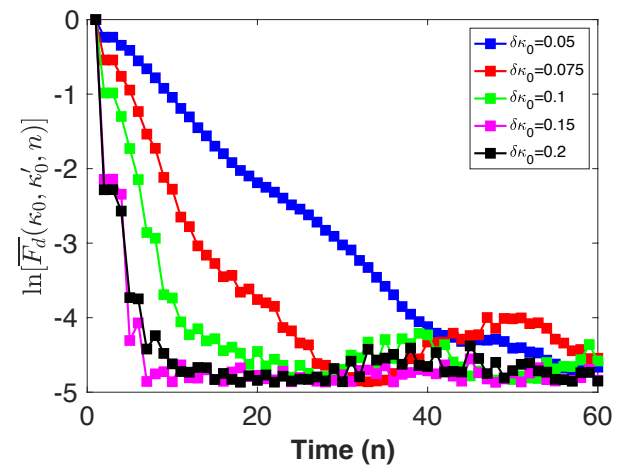

FIG. 13: Loschmidt decay on a linear log scale for some values of $\delta \kappa_{0}$ the perturbation strength. $\kappa_{0}=2 \pi$ and $j=64$. Increasing the perturbation strength results in saturation of the rate of exponential decay.

the quadratic term in the expansion of decay for $\kappa_{0}=0$ becomes non-zero starting with $n=1$.

Figure (14) interestingly shows somewhat counterintuitive behavior of the decay of Loschmidt echo with chaos. At first, it appears, more chaos leads to less echo decay for a coherent wave packet starting at $|0,0\rangle$. However, the state $|0,0\rangle$ is on a period 4 orbit and will rapidly become delocalized with support over the pe-

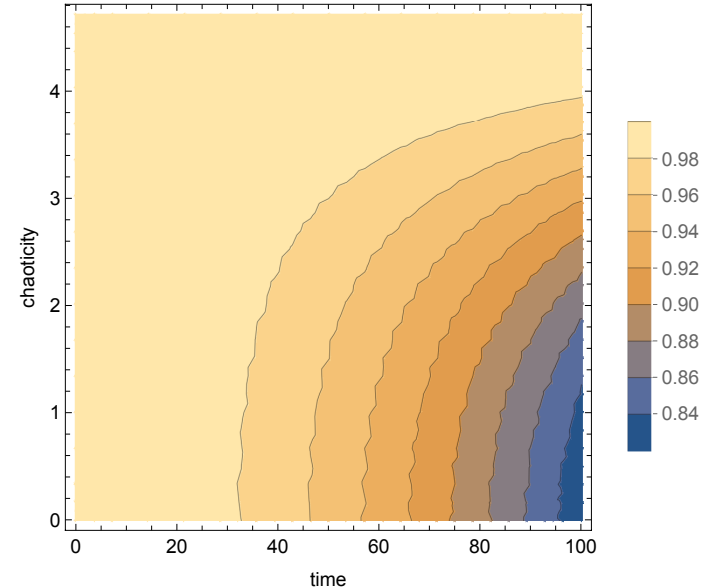

FIG. 14: Loschmidt decay for $|000\rangle$ with respect to the chaoticity parameter $\kappa_{0} \in[0,3 \pi / 2]$ and time $n$. Perturbation strength is fixed at 0.005 .

riod 4 phase space points. Fidelity decay for delocalized states having a high participation ratio is in general inversely correlated with the degree of chaos 64. As a contrast, consider the three-qubit state, $\left|\psi_{0}\right\rangle=|+++\rangle$, corresponding to a fixed point of the map, where $|+\rangle=$ $\frac{1}{\sqrt{2}}(|0\rangle+i|1\rangle)$ is an eigenvector of $\sigma_{y}$ with eigenvalue +1 . This state delocalizes when the fixed point loses stabiity and the echo decay increases with the increase of chaos $\left(\kappa_{0} \in[0,3 \pi / 2]\right)$ in the system. When the initial state is $\otimes^{3}|+\rangle_{y}=\left(\left|\phi_{1}^{+}\right\rangle+\sqrt{3} i\left|\phi_{2}^{+}\right\rangle\right) / 2$, corresponding to the coherent state at $|\pi / 2,-\pi / 2\rangle$, the evolution lies entirely in the positive parity sector. We have, $\mathcal{U}^{n}|+++\rangle_{y}$ equal to

$\left|\psi_{n}\right\rangle=\frac{1}{2} e^{-i n\left(\frac{\pi}{4}+\kappa\right)}\left[\left(\alpha_{n}-i \sqrt{3} \beta_{n}^{*}\right)\left|\phi_{1}^{+}\right\rangle+\left(\beta_{n}+i \sqrt{3} \alpha_{n}^{*}\right)\left|\phi_{2}^{+}\right\rangle\right]$.

Defining $\gamma_{n}=\left(\alpha_{n}-i \sqrt{3} \beta_{n}^{*}\right) / 2$ and $\delta_{n}=\left(\beta_{n}+i \sqrt{3} \alpha_{n}^{*}\right) / 2$, we can obtain the fidelity decay expression at time $n$ as before.

$$
\begin{aligned}
F\left(\kappa_{0}, \kappa_{0}^{\prime}, n,\left|\psi_{0}\right\rangle\right) & =\left|\left\langle+++\left|\mathcal{U}^{-n}\left(\kappa_{0}\right) \mathcal{U}^{n}\left(\kappa_{0}^{\prime}\right)\right|+++\right\rangle\right|^{2} \\
& =\left|\gamma_{n}^{*} \tilde{\gamma_{n}}+\delta_{n}^{*} \tilde{\delta_{n}}\right|^{2}
\end{aligned}
$$

where $\tilde{\gamma_{n}}$ and $\tilde{\delta_{n}}$ are $\gamma_{n}\left(\kappa_{0}^{\prime}\right)$ and $\delta_{n}\left(\kappa_{0}^{\prime}\right)$ respectively.

Decay of Loschmidt echo for the $|+++\rangle$ for small perturbations follows the quadratic decay and also increases with the increase of chaos $\left(\kappa_{0} \in[0,3 \pi / 2]\right)$ in the system. 


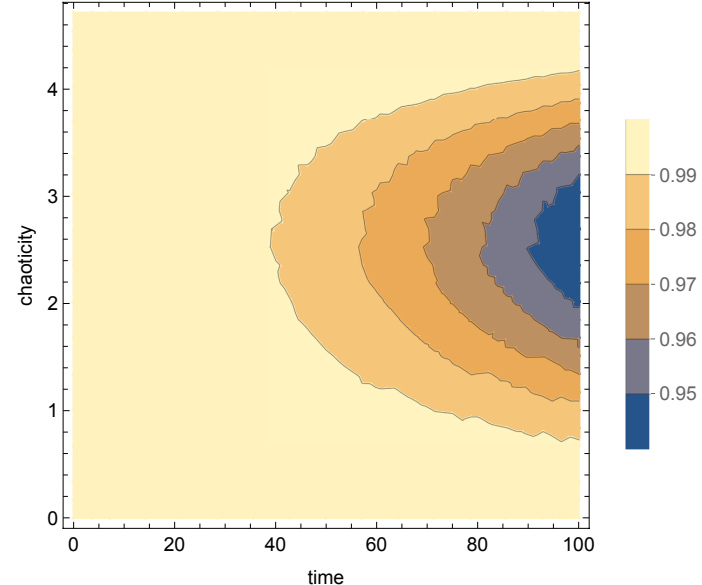

FIG. 15: Loschmidt decay for $|+++\rangle$ with respect to the chaoticity parameter $\kappa_{0} \in[0,3 \pi / 2]$ and time $n$. Perturbation strength is fixed at 0.005 .

\section{SUMMARY AND DISCUSSION}

Quantum chaos investigates the footprints of classical chaos in the quantum world. We posed an intriguing question - how deep in the quantum regime one can hope to find these signatures? In our work, we addressed this question with a provocative answer - we find signatures of classical Lyapunov exponents as captured by OTOCs even in quantum systems consisting of as few as 3 and 4 qubits. Our results for Loschmidt echo, another quantifier of chaos based on sensitive dependence of a system to perturbations in dynamics, suggest a more feeble signature of chaos for the kicked top with lower values of angular momentum. Through numerical study, we have shown that one needs to go to sufficiently high quantum numbers to see a forerunner to the exponential Lyapunov decay in the Loschmidt echo. However, for certain initial states, we do see the effects of delocalization, periodic orbits, and chaos in the decay of the echo signal in deep quantum regime of 3 and 4 qubit kicked top. How do these states fare under environmental decoherence would be an interesting future direction to explore.

Recent studies involving a related concept, the Adiabatic Guage Potential (AGP) which is the generator of adiabatic deformations between eigenstates, serves as a probe to detect chaos in systems with large Hilbert spaces [85]. An interesting direction for the future is to compare the effectiveness of AGP with that of Loschmidt echo in detecting chaos.

One interesting observation from our work was the case of $\kappa_{0}=\pi j$, the chaoticity parameter for the kicked top. As we saw, for the value of $j=2$, the Floequet operator in this case, has interesting decomposition in terms of sum of 4 pure rotations and similar sum exists for $\kappa_{0}=\pi r / s$ with $r$ and $s$ relatively prime to each other. On the one hand, this paves way for some experiments where the nonlinear twist is replaced by a sum of rotations. On the other hand, this gives us some insights into the origin of chaos and complexity in a system with a classical limit of just two degrees of freedom. It is also worth noting that it is very rare that systems exhibiting signatures of chaos are exactly solvable. A conservative system with as many constants of motion as its degrees of freedom is said to be integrable and its dynamics regular. In the quantum world, these constants of motion become operators that commute with the Hamiltonian. Lack of sufficient constants of motion leads to non-integrability and the random matrix conjecture in the quantum domain. Exactly solvable systems give us a reference to study departure from integrability and transition to chaos upon the introduction of perturbations breaking the necessary symmetries via the KAM theorem. Our work paves way for the search for more systems that are "chaotic" yet solvable. For example, a system of coupled kicked tops 86, which consists of two spins coupled via hyperfine interactions and one of them periodically kicked can be made to have connections with a many-body model considering a large spin as a collection of spin $1 / 2$ particles. We hope our work will be interesting and useful to the quantum chaos community as well as experimentalists.
[1] C. Neill, P. Roushan, M. Fang, Y. Chen, M. Kolodrubetz, Z. Chen, A. Megrant, R. Barends, B. Campbell, B. Chiaro, et al., Nature Physics 12, 1037 (2016).

[2] A. M. Kaufman, M. E. Tai, A. Lukin, M. Rispoli, R. Schittko, P. M. Preiss, and M. Greiner, Science 353, 794 (2016), ISSN 0036-8075, http://science.sciencemag.org/content/353/6301/794.full.p URL http://science.sciencemag.org/content/353/ $6301 / 794$

[3] P. A. Miller and S. Sarkar, Phys. Rev. E 60, 1542 (1999).
[4] A. Lakshminarayan, Phys. Rev. E 64, 036207 (2001).

[5] J. N. Bandyopadhyay and A. Lakshminarayan, Phys. Rev. Lett. 89, 060402 (2002).

[6] A. Tanaka, H. Fujisaki, and T. Miyadera, Phys. Rev. E 66, 045201 (2002), URL https://link.aps .org/doi/ 10.1103/PhysRevE.66.045201

df, [7] A. Lakshminarayan and V. Subrahmanyam, Phys. Rev. A 67, 052304 (2003).

[8] J. N. Bandyopadhyay and A. Lakshminarayan, Phys. Rev. E 69, 016201 (2004). 
[9] S. Ghose and B. C. Sanders, Phys. Rev. A 70, 062315 (2004).

[10] A. Lakshminarayan and V. Subrahmanyam, Phys. Rev. A 71, 062334 (2005), URL https://link.aps.org/doi/ 10.1103/PhysRevA.71.062334.

[11] C. M. Trail, V. Madhok, and I. H. Deutsch, Physical Review E 78, 046211 (2008).

[12] M. Lombardi and A. Matzkin, Phys. Rev. E 83, 016207 (2011), URL https://link.aps.org/doi/10. 1103/PhysRevE.83.016207

[13] V. Madhok, C. A. Riofrío, S. Ghose, and I. H. Deutsch, Phys. Rev. Lett. 112, 014102 (2014).

[14] V. Madhok, V. Gupta, D.-A. Trottier, and S. Ghose, Physical Review E 91, 032906 (2015).

[15] V. Madhok, S. Dogra, and A. Lakshminarayan, Optics Communications 420, 189 (2018), ISSN 00304018, URL https://www.sciencedirect.com/science/ article/pii/S0030401818302542.

[16] A. Piga, M. Lewenstein, and J. Q. Quach, Phys. Rev. E 99, 032213 (2019), URL https://link.aps.org/doi/ 10.1103/PhysRevE.99.032213.

[17] M. Kumari and S. Ghose, Phys. Rev. A 99, 042311 (2019), URL https://link.aps.org/doi/10. 1103/PhysRevA.99.042311

[18] A. Lerose and S. Pappalardi, Phys. Rev. A 102, 032404 (2020), URL https://link.aps.org/doi/10. 1103/PhysRevA.102.032404

[19] S. Chaudhury, A. Smith, B. E. Anderson, S. Ghose, and P. S. Jessen, Nature 461, 768 (2009).

[20] F. Haake, Quantum Signatures of Chaos (Spring-Verlag, Berlin, 1991).

[21] S. Dogra, V. Madhok, and A. Lakshminarayan, Phys. Rev. E 99, 062217 (2019), URL https://link.aps.org/ doi/10.1103/PhysRevE.99.062217

[22] J. B. Ruebeck, J. Lin, and A. K. Pattanayak, Phys. Rev. E 95, 062222 (2017).

[23] U. T. Bhosale and M. S. Santhanam, Phys. Rev. E 98, 052228 (2018), URL https://link.aps.org/doi/ 10.1103/PhysRevE.98.052228.

[24] V. R. Krithika, V. S. Anjusha, U. T. Bhosale, and T. S. Mahesh, Phys. Rev. E 99, 032219 (2019), URL https: //link.aps.org/doi/10.1103/PhysRevE.99.032219

[25] A. Peres, Phys. Rev. A 30, 1610 (1984).

[26] R. Schack and C. M. Caves, Phys. Rev. E 53, 3257 (1996).

[27] M. Berry and M. Tabor, Proceedings of the Royal Society of London A: Mathematical, Physical and Engineering Sciences 356, 375 (1977), ISSN 0080-4630.

[28] O. Bohigas and J. Flores, Physics Letters B 35, 383 (1971).

[29] M. V. Berry, Journal of Physics A: Mathematical and General 10, 2083 (1977), URL http://stacks .iop.org/ $0305-4470 / 10 / i=12 / a=016$.

[30] M. Berry, N. Balazs, M. Tabor, and A. Voros, Annals of Physics 122, 26 (1979), ISSN 00034916, URL http://www.sciencedirect.com/science/ article/pii/0003491679902963.

[31] A. Voros, Ann. Inst. Henri Poincaré A 24, 31 (1976).

[32] E. J. Heller, Physical Review Letters 53, 1515 (1984).

[33] S. W. McDonald and A. N. Kaufman, Phys. Rev. Lett. 42, 1189 (1979), URL https://link.aps.org/doi/10. 1103/PhysRevLett.42.1189

[34] M. Robnik and M. V. Berry, Journal of Physics A: Mathematical and General 18, 1361 (1985).
[35] M. C. Gutzwiller, Journal of Mathematical Physics 12, 343 (1971).

[36] B. Swingle, G. Bentsen, M. Schleier-Smith, and P. Hayden, Physical Review A 94, 040302 (2016).

[37] P. Hayden and J. Preskill, Journal of High Energy Physics 2007, 120 (2007).

[38] J. Maldacena and D. Stanford, Phys. Rev. D 94, 106002 (2016), URL https://link.aps.org/doi/10. 1103/PhysRevD.94.106002.

[39] J. Maldacena, S. H. Shenker, and D. Stanford, Journal of High Energy Physics 2016, 106 (2016), ISSN 1029-8479, URL https : //doi .org/10.1007/JHEP08(2016) 106.

[40] T. Hartman and J. Maldacena, Journal of High Energy Physics 2013, 14 (2013).

[41] S. H. Shenker and D. Stanford, Journal of High Energy Physics 2014, 67 (2014).

[42] Y. Sekino and L. Susskind, Journal of High Energy Physics 2008, 065 (2008).

[43] N. Lashkari, D. Stanford, M. Hastings, T. Osborne, and P. Hayden, Journal of High Energy Physics 2013, 22 (2013), ISSN 1029-8479, URL https://doi.org/10. 1007/JHEP04(2013)022.

[44] P. Hosur, X.-L. Qi, D. A. Roberts, and B. Yoshida, Journal of High Energy Physics 2016, 4 (2016).

[45] E. Iyoda and T. Sagawa, arXiv:1704.04850 (2017).

[46] A. I. Larkin and Y. N. Ovchinnikov, JETP 28, 1200 (1969).

[47] J. S. Cotler, D. Ding, and G. R. Penington, Ann. Phys. 396, 318 (2018), ISSN 0003-4916, URL http://www.sciencedirect.com/science/article/ pii/S000349161830191X

[48] K. Hashimoto, K. Murata, and R. Yoshii, J. High Energy Phys. 2017, 138 (2017), ISSN 1029-8479.

[49] B. Swingle, Nature Physics 14, 988 (2018).

[50] J. Chávez-Carlos, B. López-del Carpio, M. A. Bastarrachea-Magnani, P. Stránský, S. LermaHernández, L. F. Santos, and J. G. Hirsch, Phys. Rev. Lett. 122, 024101 (2019), URL https://link. aps.org/doi/10.1103/PhysRevLett.122.024101.

[51] E. B. Rozenbaum, S. Ganeshan, and V. Galitski, Phys. Rev. Lett. 118, 086801 (2017), URL https://link.aps . org/doi/10.1103/PhysRevLett.118.086801

[52] A. Lakshminarayan, Phys. Rev. E 99, 012201 (2019), URL https://link.aps.org/doi/10.1103/PhysRevE. 99.012201

[53] I. García-Mata, M. Saraceno, R. A. Jalabert, A. J. Roncaglia, and D. A. Wisniacki, Phys. Rev. Lett. 121, 210601 (2018), URL https://link.aps.org/doi/10. 1103/PhysRevLett.121.210601.

[54] S. Moudgalya, T. Devakul, C. W. von Keyserlingk, and S. L. Sondhi, Phys. Rev. B 99, 094312 (2019), URL https://link.aps.org/doi/10.1103/PhysRevB. 99.094312

[55] J. Rammensee, J. D. Urbina, and K. Richter, Phys. Rev. Lett. 121, 124101 (2018), URL https://link .aps.org/ doi/10.1103/PhysRevLett.121.124101.

[56] R. A. Jalabert, I. García-Mata, and D. A. Wisniacki, Phys. Rev. E 98, 062218 (2018), URL https://link. aps.org/doi/10.1103/PhysRevE.98.062218

[57] R. Prakash and A. Lakshminarayan, Phys. Rev. B 101, 121108 (2020), URL https://link.aps.org/doi/10. 1103/PhysRevB.101.121108

[58] A. Seshadri, V. Madhok, and A. Lakshminarayan, Phys. Rev. E 98, 052205 (2018), URL https ://link .aps .org/ 
doi/10.1103/PhysRevE.98.052205

[59] L. M. Sieberer, T. Olsacher, A. Elben, M. Heyl, P. Hauke, F. Haake, and P. Zoller, npj Quantum Information 5, 1 (2019).

[60] C. Yin and A. Lucas, Quantum operator growth bounds for kicked tops and semiclassical spin chains (2020), 2010.06592.

[61] A. Peres, Quantum theory: concepts and methods, vol. 57 (Springer Science \& Business Media, 2006).

[62] A. Peres, Physical Review A 30, 1610 (1984).

[63] T. Prosen, T. H. Seligman, and M. Žnidarič, Progress of Theoretical Physics Supplement 150, 200 (2003).

[64] T. Gorin, T. Prosen, T. H. Seligman, and M. Znidarič, Physics Reports 435, 33 (2006).

[65] R. Sankaranarayanan and A. Lakshminarayan, Phys. Rev. E 68, 036216 (2003), URL https : //link . aps .org/ doi/10.1103/PhysRevE.68.036216

[66] W. H. Zurek and J. P. Paz, Phys. Rev. Lett. 72, 2508 (1994).

[67] B. Georgeot and D. L. Shepelyansky, Phys. Rev. E 62, 3504 (2000), URL https://link.aps.org/doi/10. 1103/PhysRevE.62.3504

[68] B. Georgeot and D. L. Shepelyansky, Phys. Rev. E 62, 6366 (2000), URL https://link.aps.org/doi/10. 1103/PhysRevE.62.6366

[69] P. Hauke, F. M. Cucchietti, L. Tagliacozzo, I. Deutsch, and M. Lewenstein, Reports on Progress in Physics 75, 082401 (2012).

[70] I. M. Georgescu, S. Ashhab, and F. Nori, Reviews of Modern Physics 86, 153 (2014).

[71] I. H. Deutsch, arXiv preprint arXiv:2010.10283 (2020).

[72] L. J. Fiderer and D. Braun, Nature communications 9, 1 (2018).
[73] M. Kuś, R. Scharf, and F. Haake, Zeitschrift für Physik B Condensed Matter 66, 129 (1987).

[74] A. Peres, Quantum Theory: Concepts and Methods (Kluwer Academic Publishers, New York, 2002).

[75] G. J. Milburn, Simulating nonlinear spin models in an ion trap (1999), arXiv:quant-ph/9908037.

[76] X. Wang, S. Ghose, B. C. Sanders, and B. Hu, Phys. Rev. E 70, 016217 (2004).

[77] T. Prosen, Progress of Theoretical Physics Supplement 139, 191 (2000).

[78] B. Yan, L. Cincio, and W. H. Zurek, arXiv e-prints arXiv:1903.02651 (2019), 1903.02651.

[79] S. Omanakuttan and A. Lakshminarayan, Phys. Rev. E 99, 062128 (2019), URL https://link.aps.org/doi/ 10.1103/PhysRevE.99.062128

[80] R. Prakash and A. Lakshminarayan, Acta Phys. Polon. A 136, 803 (2019), 1911.02829.

[81] I. García-Mata, A. J. Roncaglia, and D. A. Wisniacki, Philosophical Transactions of the Royal Society A: Mathematical, Physical and Engineering Sciences 374, 20150157 (2016).

[82] P. Zanardi and D. A. Lidar, Physical Review A 70, 012315 (2004).

[83] R. J. Glauber and F. Haake, Phys. Rev. A 13, 357 (1976).

[84] R. R. Puri, Mathematical Methods of Quantum Optics (Springer, Berlin, 2001).

[85] M. Pandey, P. W. Claeys, D. K. Campbell, A. Polkovnikov, and D. Sels, Phys. Rev. X 10, 041017 (2020), URL https://link.aps.org/doi/10. 1103/PhysRevX.10.041017

[86] C. M. Trail, V. Madhok, and I. H. Deutsch, Phys. Rev. E 78, 046211 (2008). 